Published in final edited form as:

Nat Microbiol. 2019 December ; 4(12): 2101-2108. doi:10.1038/s41564-019-0603-6.

\title{
In vivo structure of the Legionella type II secretion system by electron cryotomography
}

\author{
Debnath Ghosal ${ }^{1}$, Ki Woo Kim ${ }^{1,2}$, Huaixin Zheng ${ }^{3,4}$, Mohammed Kaplan ${ }^{1}$, Hilary K. \\ Truchan $^{3}$, Alberto E. Lopez ${ }^{3}$, lan E. McIntire ${ }^{3}$, Joseph P. Vogel ${ }^{5}$, Nicholas P. Cianciotto ${ }^{3}$, \\ Grant J. Jensen ${ }^{1,6, *}$ \\ ${ }^{1}$ Division of Biology and Biological Engineering, California Institute of Technology, Pasadena, CA \\ 91125, USA. \\ ${ }^{2}$ School of Ecology and Environmental System, Kyungpook National University, Sangju, 37224 \\ Korea \\ ${ }^{3}$ Department of Microbiology and Immunology, Feinberg School of Medicine, Northwestern \\ University, Chicago, IL 60611, USA. \\ ${ }^{4}$ Current Address: Department of Immunology, School of Basic Medical Sciences, Zhengzhou \\ University, Zhengzhou City, Henan Province 450000, China \\ 5 Department of Molecular Microbiology, Washington University School of Medicine, St. Louis, MO \\ 63110, USA. \\ ${ }^{6}$ Howard Hughes Medical Institute, Pasadena, CA 91125, USA.
}

\section{Abstract}

The type II secretion system (T2SS) is a multiprotein envelope-spanning assembly that translocates a wide range of virulence factors, enzymes and effectors through the outer membrane $(\mathrm{OM})$ of many Gram-negative bacteria ${ }^{1-3}$. Here, using electron cryotomography (ECT) and subtomogram averaging methods, we reveal the in vivo structure of an intact T2SS, imaged within the human pathogen Legionella pneumophila. Although the T2SS has only limited sequence and component homology with the evolutionarily-related type IV pilus (T4P) system ${ }^{4,5}$, we show that their overall architectures are remarkably similar. Despite similarities, there are also differences, including for instance that the T2SS-ATPase complex is usually present but disengaged from the inner membrane (IM), the T2SS has a much longer periplasmic vestibule, and it has a short-lived flexible pseudopilus. Placing atomic models of the components into our ECT map produced a

\footnotetext{
Users may view, print, copy, and download text and data-mine the content in such documents, for the purposes of academic research, subject always to the full Conditions of use:http://www.nature.com/authors/editorial_policies/license.html\#terms

*Correspondence: jensen@caltech.edu.

Contributions: D.G. and G.J.J. conceived the project. D.G. prepared samples, recorded and processed tomography data. D.G., K.W.K. and G.J.J. analyzed data. H.Z., H.K.T., A.E.L., I.E.M., N.P.C. and J.P.V. generated and characterized L. pneumophila mutants. M.K. helped in model building. D.G., K.W.K., N.P.C. and G.J.J. wrote the manuscript with input from all the authors.

Competing Interests: The authors declare no competing interests.

Data availability

The subtomogram average of the L. pneumophila T2SS has been deposited in the Electron Microscopy Data Bank under the following accession codes: EMD-20713 (wild-type aligned on the OM part); EMD-20712 (wild-type, aligned on the IM part). All additional data/information are available from the authors upon request. The authors declare that all data supporting the findings of this study are available within the paper and its supplementary information documents.
} 
complete architectural model of the intact T2SS that provides insights into the structure and function of its components, its position within the cell envelope, and the interactions between its different subcomplexes.

Evolutionarily-related to bacterial type IV pili ${ }^{4,5}$, canonical T2SS are mainly distributed among genera of Proteobacteria, including representatives within $\alpha_{-}, \beta_{-}, \gamma_{-}$, and $\delta_{-}$ proteobacteria ${ }^{3,6,7}$ The T2SS apparatus generally employs 12 "core" components, which, for simplicity, will be referred to here as T2S C, D, E, F, G, H, I, J, K, L, M, and $\mathrm{O}^{7,8}$. These components have been described as being part of four subcomplexes ${ }^{7,8}$. The first subcomplex is a multimer of the T2S D protein that provides the ultimate portal or gate for substrate transit across the $\mathrm{OM}$ and out of the cell; i.e., the so-called secretin ${ }^{9}$. Interacting with the secretin to create a periplasm-spanning channel is an IM-anchored subcomplex or platform comprised of $\mathrm{T} 2 \mathrm{~S} \mathrm{~F}, \mathrm{~L}$, and $\mathrm{M}^{10}$. The OM-associated subcomplex and the IMassociated subcomplexes are coupled by the IM-associated "clamp protein" $\mathrm{T} 2 \mathrm{~S} \mathrm{C}^{10}$. The third subcomplex is a pseudopilus that consists of the major pseudopilin T2S G and minor pseudopilins T2S H, I, J, and $\mathrm{K}^{10}$. The pseudopilus is thought to span the periplasm within the channel created by the interaction of the IM platform with the secretin and is believed to act in a "piston-" or "screw-like" fashion to drive substrate through the OM portal",11,12. The fourth subcomplex is a hexamer of T2S E, a cytoplasmic ATPase that is recruited to the IM in order to "power" the secretion process ${ }^{10}$. The T2S O protein is an IM- prepilin peptidase ${ }^{7,8}$.

In vitro structural studies of purified components and subcomplexes have significantly improved our understanding of the T2SS ${ }^{13-26}$. Despite these advances, it is not clear how the different components of the T2SS are positioned and interact with each other within an intact cell envelope (in situ).

ECT has recently emerged as a powerful tool to investigate macromolecular complexes in situ at nanometer resolution ${ }^{27-31}$. To reveal the intact structure of the bacterial T2SS in situ, we used ECT and image nearly 2000 frozen-hydrated L. pneumophila cells (Supplementary Table 1). In our tomograms, we observed multiple electron dense "hour-glass" shaped particles (Fig. 1A-D), reminiscent of the secretin structure", in the periplasm. These structures were primarily localized in the vicinity of cell poles and were not associated with any exocellular filaments (Fig. 1A, B). We also observed several top views of these particles with a diameter $\sim 10 \mathrm{~nm}$ (Fig. 1E, F). While $L$. pneumophila cells encode for a type IVa pilus (T4aP) system, which also has a secretin ${ }^{32}$, several lines of evidence suggest that all the structures we saw were T2SSs. First, in our 2000 tomograms, we did not see any T4aP filaments coming out of the L. pneumophila cells, suggesting that under our growth conditions, $\mathrm{T} 4 \mathrm{aP}$ is not expressed. This is consistent with the earlier observation in which $30^{\circ} \mathrm{C}$ was found to be better than $37^{\circ} \mathrm{C}$ for the assembly of the T4aP systems ${ }^{32}$. Second, no particles were visible in a strain lacking $L$. pneumophila T2SS components T2S D and E $(\Delta l s p D E)$ (Fig. 1G), but they were still present in a strain lacking the T4aP secretin $(\Delta p$ ilQ) (Fig. 1H). In a double deletion strain lacking the T2SS secretin as well as the T4aP secretin $(\Delta l s p D E, \Delta$ pilQ), again no particles were visible (Fig. 1I, Supplementary Table 1). Finally, our analysis of the T2SS secretin revealed distinct features as compared to the T4aP secretin 
(see Extended Data Fig. 1 and discussion below), confirming that the "hour-glass" shaped particles are L. pneumophila T2SSs.

To reveal the in situ molecular architecture of the intact $L$. pneumophila T2SS, we generated a subtomogram average using 440 particles. Our analysis showed substantial flexibility between the OM-associated secretin complex and the rest (Extended Data Fig. 2A). To overcome this, we performed focused alignment on the OM- and IM-associated subcomplexes separately and produced a composite structure (Fig. 1J, Extended Data Fig. $2 \mathrm{~A}-\mathrm{C}$ ) which had a variable local resolution between $\sim 2.5-5.5 \mathrm{~nm}$ as estimated by ResMap (Extended Data Fig. 2D). The final average revealed that the T2SS is composed of a $\sim 23 \mathrm{~nm}$ long vestibule that reaches down most of the way through the periplasm and opens up in the OM with an $\sim 8 \mathrm{~nm}$ wide pore (Fig. 1J). There are two distinct densities in the lumen of the secretin channel near the two ends: (i) a "gate" just underneath the OM pore and (ii) a previously unreported structure, "plug", near the base (Extended Data Fig. 2E). Although the gate structure resolved well in our subtomogram average, the plug structure was poorly visible. Further analysis revealed that the plug is either not present or so dynamic in a subset of the particles that it is almost invisible in the final average (Extended Data Fig. 2E). Below the secretin channel, $7.5 \mathrm{~nm}$ away from the IM, there is a lower- periplasmic ring and a short stem at the same level (Fig. 1J). The lower-periplasmic ring is $17 \mathrm{~nm}$ wide in diameter (peak to peak) and the stem structure is in the middle of this ring (Fig. 1J). On the cytoplasmic side, there are three distinct densities: a dome-like density immediately below the IM (cytoplasmic dome), a ring-like density of diameter $20 \mathrm{~nm}$ (peak to peak) (cytoplasmic ring) surrounding the dome and finally, $13 \mathrm{~nm}$ away from the IM a 12-nm wide (peak to peak) cytoplasmic disk (Fig. 1J). A comparison between the subtomogram averages of the T2SS and the non-piliated in situ structures of the Myxococcus xanthus T4aP (MxT4aP) and the Vibrio cholerae toxin-coregulated (TCP) type IVb pilus (VcT4bP) ${ }^{33,34}$ machines revealed that despite varied gene organization and limited component homology, the overall architectures of these three classes of molecular machines are strikingly similar (Fig. 1J-L, note cytoplasmic disks for the MxT4aP and VcT4bP are seen in other states. See Extended Data Fig. 2F).

Intriguingly, the L. pneumophila secretin (T2S D, aa $=791)$ sequence is shorter than the MxT4aP secretin (PilQ, aa $=901$ ), but in the subtomogram averages, the L. pneumophila T2SS secretin channel is $\sim 1.5$ times longer than the MxT4aP. To rationalize this, we predicted domain architectures of secretins of the L. pneumophila T2SS (T2S D or LspD), VcT4bP (TcpC) and MxT4aP (PilQ) systems using Motif-search, Phyre and the CD-vist programs $^{35,36}$ (Fig. 1M). Our analysis revealed that all three secretins (LpT2S D, MxPilQ and $\mathrm{VcTcpC}$ ) have conserved $\mathrm{C}$ - terminal secretin domains. However, preceding the secretin domain, TcpC and PilQ have only one N-domain, but T2S D has four (N3, N2, N1 and N0) (Fig. 1M, Extended Data Fig. 3A). N- domains are known to fold into rigid structures and thus resolved well in the subtomogram average ${ }^{33,37}$. In contrast, the N-terminal AMIN domains of PilQ are not ordered and thus remained indiscernible in the subtomogram average.

In our subtomogram average, we saw densities for the cytoplasmic dome, ring and disk of the T2SS (Fig. 1J). The cytoplasmic dome is also visible in the piliated as well as in the non- 
piliated state of the MxT4aP and VcT4bP machines (Fig. 1K,L, Extended Data Fig. 2F), but the cytoplasmic ring and the disk are only visible in certain states of the MxT4aP and $V c T c p C$ systems interpreted previously as assembly states (Fig. 1J-L, Extended Data Fig. $2 \mathrm{~F})$.

Our in situ structure revealed how the T2SS is positioned in the cell envelope with respect to the OM, peptidoglycan (PG) and IM. In our average, the secretin complex forms an OMspanning pore. However, when we aligned available cryoEM structures of assembled secretin channels onto our density map based on the position of the gate (Extended Data Fig. $3 \mathrm{~A}-\mathrm{C}$ ), the tip of the secretin penetrated only the inner leaflet of the OM, suggesting secretin assemblies might have a different, more extended conformation in vivo, and collapse or change conformation during detergent extraction and purification.

To investigate if the T2SS is activated under the growth conditions used, we looked for T2SS- effector release in the L. pneumophila culture supernatant. Western blot analysis of the L. pneumophila culture supernatant showed consistent presence of the effectors CelA, ProA and LegP during mid-log phase and late-log phase, suggesting that at least some of the T2SSs are active (Fig. 2P). This effector release is T2SS-specific because a T2SSnonfunctional mutant $(\Delta / s p D E)$ did not release any of these effectors (Fig. 2P). Interestingly, in a strain lacking the functional Dot/Icm type IV secretion system $(\Delta d o t / i c m)$, we found $25 \%$ more T2SS particles. However, this does not correlate with an increase in T2SS effector release (Fig. 2P).

In the T2SS, four minor pseudopilins (T2S I, J, K and H) and a major pseudopilin (T2S G) constitute a pseudopilus that is thought to act as a piston to extrude exoproteins through the $\mathrm{T}^{2} \mathrm{SS}^{7,11,12}$. In mutant strains lacking the pseudopilins, the activity of the T2SS is severely impaired $^{38}$. Since we did not see a pseudopilus in our subtomogram average (Fig. 1J), we carefully looked through all the individual T2SS particles in our tomograms and found that a fraction $(\sim 20 \%)$ of the particles had density likely from pseudopili (Fig. 2A-E). In these particles, the putative pseudopili extend $(\sim 14 \mathrm{~nm})$ from the IM to the base of the T2S D channel. The positions of these densities are variable, however, so they remain invisible in the average. To confirm the identity of these pseudopili-like densities, we imaged a $L$. pneumophila mutant strain lacking all the minor pseudopilins $(\Delta l s p H I J K)$ and another one lacking all the pseudopilins $(\Delta I s p G H I J K)$. No pseudopilus-like density was visible associated with the T2SS particles in any of these mutant strains confirming that the rod-like densities just below the secretin channel are indeed pseudopili (Extended Data Fig. 3D).

In our tomograms, the lower-periplasmic ring densities in individual particles were just as visible as the secretin density, but their positions varied between particles, explaining why this density resolved poorly in the subtomogram average (Fig. 2F-J). Furthermore, there were also many particles with just the periplasmic vestibule and without a detectable pseudopilus or a lower- periplasmic ring (Fig. 2K-O). These particles are likely either assembly or disassembly intermediates.

Our in situ structure of the T2SS showed that the basic architectures of the MxT4aP, VcT4bP and LpT2SS are clearly very similar (Fig. 1J-L, Extended Data Fig. 1). There are 
also many interesting differences, however, which lead to specific hypotheses about the roles and adaptations of many of the components as discussed below (Extended Data Fig. 1).

The T2SS has a much longer periplasmic vestibule $(23 \mathrm{~nm})$ than the T4aP and T4bP systems $(15 \mathrm{~nm})$. It is unclear if this difference is because the T2SS translocates dozens of exoproteins through the secretin channel, whereas the $\mathrm{T} 4 \mathrm{aP}$ and $\mathrm{T} 4 \mathrm{bP}$ systems primarily secrete their pili. In our structure, we observed a poorly-resolved plug density near the base of the secretin vestibule (Extended Data Fig. 2E). T2S C is known to interact with the NO domain of T2S D through its HR domain and recruit T2SS effectors into the complex ${ }^{22}$. Therefore, the plug density could be the HR domain of T2S $\mathrm{C}^{22}$ or, perhaps cargo that is waiting to be extruded by the pseudopilus.

Unlike theMxT4aP and the VcT4bP systems, the L. pneumophila T2SS does not encode a pilotin $^{3,39}$. Although the T2S D sequence has a pilotin binding S-domain, no putative pilotin sequence was found in the $L$. pneumophila genome. This is consistent with the idea that pilotins are not essential for all secretin pores to assemble. It remains unclear how the $L$. pneumophila T2S D is targeted to the cell pole and associates with the PG. In the MxT4aP system, the N-terminal AMIN domains of PilQ tether the T4aP machine to the PG layer ${ }^{34}$. In our L. pneumophila tomograms, the PG layer is located immediately beneath the OM (Extended Data Fig. 4) but the putative PG binding domain of T2S D is N-terminal to the NO domain, which is $\sim 20 \mathrm{~nm}$ below the PG (Fig. 1M). One possibility is that the linker between the NO domain and the putative PG binding domain bridges this gap. Curiously, the L. pneumophila T2SS also lacks other PG binding complexes like those present in Vibrio spp., Aeromonas spp. and in E. coli T2SS (e.g. gspAB homologs) ${ }^{3,40}$.

In the L. pneumophila T2SS, the periplasmic domains of T2S L and $\mathrm{M}$ form a lowerperiplasmic ring similar to those present in the MxT4aP and VcT4bP systems. PilO and PilN (the two proteins that form the lower-periplasmic ring in the MxT4aP), and T2S L and T2S $\mathrm{M}$ all begin with an $\mathrm{N}$-terminal transmembrane helix, followed by a ferredoxin-like fold in the periplasm. Previously, it was hypothesized that in the MxT4aP, the lower-periplasmic ring likely transmits information about the status of the pilus to the cytoplasmicsubcomplex ${ }^{34}$. Given that T2S L and T2S M have recently been shown to interact with secreted effectors ${ }^{41}$, T2S L and M may likewise sense cargo in the periplasm and signal the cytoplasmic complex to extend the pseudopilus. Signal sensing and transmission are likely simpler in the T2SS, however, since while the lower-periplasmic ring in the MxT4aP is composed of 12 or more copies of both PilO and PilN, in L. pneumophila, the lowerperiplasmic ring is apparently comprised of only six copies each of T2S L and M. Based on these stoichiometries, the conservation of fold, and the appearances of the rings in the subtomogram averages, we predict that the basic structures of these lower-periplasmic rings are the same, but there are two closely-packed rings in the MxT4aP, as opposed to only one in the T2SS. Signaling likely involve transitions between homodimer and heterodimer associations, as the presence of cargo biases the interactions between T2S L and M towards heterodimers ${ }^{41}$, and the VcT4bP system, which may not signal (the pilus is never retracted by an ATPase), has only a single lower-periplasmic-ring protein $(\mathrm{TcpD})^{33}$. It remains elusive how the symmetry mismatch between the secretin channel, T2S C and the lower-periplasmic 
ring is coordinated and what the role of symmetry mismatch is in effector loading and export.

The conservation of transmembrane helices in all the lower-periplasmic-ring proteins suggests an important role in signal transmission or structure, but the number of helices present and their connections to the cytoplasmic ring vary. Assuming there are six copies of both T2S L and M, a ring of 12 transmembrane helices will be present in the IM. This contrasts with the 24 or more helices from PilN and PilO in the MxT4aP. Perhaps in partial compensation, the T2SS clamp protein T2S C begins with a transmembrane helix, adding 612 more helices to the ring (according to current estimates of stoichiometry), while its counterparts in the MxT4aP and FcT4bP systems (PilP and TcpS, respectively) are simply lipoproteins. Curiously, in the FcT4bP system, there are again two proteins contributing to alpha helices in the IM, but in this case one is from the periplasmic ring (TcpD) and the other is from the cytoplasmic ring (TcpR). The L. pneumophila T2S exhibits yet another variation, in that one lower-periplasmic-ring protein (T2S L) forms part of the cytoplasmic ring, with the transmembrane helix in between. We speculate that the fusion allows another adaptation, which is that in $L$. pneumophila the cytoplasmic domain of T2S L appears to not form a continuous ring. Being fused to the periplasmic ring may be important to hold it in place. Overall, seemingly a network of 18 or more interacting transmembrane helices in the IM mediate signal transmission between the lower-periplasmic ring and the cytoplasmic ring.

Just as in the MxT4aP and FcT4bP systems, the T2SS ATPase sits directly below the IM on the machine's axis, however, is positioned further away from the IM and is less well resolved. We speculate that as the T2SS rapidly switches from extending to retracting the pseudopilus (the piston model), it does so by moving the ATPase up against the adaptor T2S $\mathrm{F}$ to extend the pseudopilus, and then dropping it away from $\mathrm{T} 2 \mathrm{~S} \mathrm{~F}$ to let the pseudopilus retract. Our interpretation of the data is that most of our particles were in the retraction state, with the ATPase disengaged from T2S F. This mechanism is of course different than the MxT4aP system that has two (extension and retraction) ATPases ${ }^{34}$ In the MxT4aP case, the two ATPases are sequentially exchanged and were seen in contact with the adaptor in the cryotomograms, suggesting they are held there continuously for long periods of time. In contrast, the T2SS likely evolved to rapidly switch between short bursts of extension and retraction, and so it does not release its ATPase completely - it appears to merely "clutch" it on and off. In the retraction state, we propose that the N1E domain is close to the IM and interacts with the cytoplasmic domains of T2S L and T2S $\mathrm{F}^{17,20,42-44}$, while the N2E and CTE domains dangle on flexible linkers, causing them to resolve poorly.

In our tomograms, several individual particles exhibit putative pseudopili. No pseudopilus was seen to extend up to the gate, suggesting either the pseudopilus only pushes exoproteins into the periplasmic vestibule or an extended form of a pseudopilus (reaching up to the gate or beyond) is short-lived. Our model showed that only $\sim 5$ copies of the major pseudopilin T2S G and one copy of each of the minor pseudopilins (T2S I, J, K, H) is sufficient to span the distance between the IM and the base of the secretin. We also found that $12-15$ copies of the major pseudopilin and one copy of each of the minor pseudopilins T2S I, J, K, H would be required for the pseudopilus to extend to the secretin gate. It is not clear how the 
length of the pseudopilus is controlled. Intriguingly, the C-terminal domain of T2S L has sequence similarity (Phyre: 52 amino acids, 68\% confidence and 15\% identity) to the Cterminal domain of FliK that controls flagellar hook length ${ }^{45}$. Thus, T2S L may have multiple functions.

Overall, this study highlights commonalities and key differences between the evolutionarilyrelated $\mathrm{T} 2 \mathrm{SS}, \mathrm{T} 4 \mathrm{aP}$ and $\mathrm{T} 4 \mathrm{bP}$ machines and provides insights into its structure and function.

\section{Materials and Methods}

\section{Strains, growth conditions, and mutant generation}

All experiments were performed using the L. pneumophila Lp02 strain (thyA hsdR rpsL), a derivative of the clinical isolate L. pneumophila Philadelphia-1. Cells were grown as described previously ${ }^{27,46}$. Briefly, cells were grown in ACES buffered yeast extract (AYE) broth or on buffered charcoal yeast extract (CYE) plates. The culture media were always supplemented with thymidine $(100 \mathrm{pg} / \mathrm{ml})$, ferric nitrate and cysteine hydrochloride.

A T4SS mutant of Lp02 that lacks all 26 of the dot/icm genes (i.e., JV4044) was previously $\operatorname{described}^{47,48}$, as was a mutant of Lp02 that lacks $\operatorname{dot} H$, $\operatorname{dot} G$, and $\operatorname{dot} F\left(\right.$ i.e., JV7058) ${ }^{48}$. To generate a IspDE mutant lacking the T2SS (i.e., strain NU438) and apilQ mutant lacking T4P (i.e., strain NU439) of L. pneumophila, previously reported plasmids were introduced into Lp02 by natural transformation and then the desired mutations were introduced into the bacterial chromosome via allelic exchange ${ }^{49}$. Plasmid pOE4Kan was used to make the IspDE mutant, and pGQ::Gm was employed for making the pilQ mutant ${ }^{50,51}$. In a similar way, an IspDE pilQ double mutant lacking both T4SS and T4P (i.e., strain NU440) was constructed by introducing pGQ::Gm into strain NU438.

To generate an IspHIJK mutant lacking the minor pseudopilins (i.e., strain NU442) and an IspGHIJK mutant lacking both the major and minor pseudopilins (i.e., strain NU444) a form of allelic exchange was used. First, mutagenized alleles were generated using overlap extension PCR. The 5' and 3' flanking regions of $1 s p H I J K$ and IspGHIJK were PCR amplified from L. pneumophila 130b DNA. Then the kanamycin-resistance cassette flanked by Flp recombination target sites was PCR amplified from pKD4. We performed two-step overlap extension PCR to combine the 5' and 3' regions of IspHIJK and IspGHIJK with the respective resistance cassettes. PCR products corresponding to the correct sizes were gel purified and ligated into pGEM-T Easy (Promega) to yield either pGlspHIJK::Kn or pGlspGHIJK::Kn. These plasmids were then introduced into strain Lp02 by natural transformation and mutants were obtained by plating on CYE agar containing kanamycin and verified by PCR.

\section{Assay for secreted proteins}

L. pneumophila strains were grown in AYE broth at $37^{\circ} \mathrm{C}$ to either mid-log or late-log phase, at which times the OD660 was measured and the cultures were centrifuged. The resulting supernatants were filtered through a $0.2 \mu \mathrm{m}$ syringe filter. The supernatant and pellets were diluted in PBS and 2X Laemmli buffer according to the OD660 to normalize for bacterial numbers. The samples were subjected to SDS-PAGE and then analyzed by immunoblot as 
previously described ${ }^{52}$. Briefly, after blocking in $5 \%$ milk for $1 \mathrm{~h}$, the blots were incubated overnight with 1:5,000 dilutions of rabbit anti-CelA, anti-ProA, anti-ICDH or 1:1,000 dilutions of anti-LegP antibodies, washed, and then incubated for $1 \mathrm{~h}$ with 1:1,000 dilution of secondary HRP-conjugated goat anti-rabbit IgG antibody (Cell Signaling Technology). Images of the immunoblots were developed with ECL ${ }^{\mathrm{TM}}$ Western Blotting Detection Reagent (GE Healthcare).

\section{Sample preparation for electron cryotomography}

L. pneumophila Lp02 cells were grown till early stationary stage $\left(\mathrm{OD}_{600} \sim 2.8\right)$ and harvested. Cells were mixed with 10-nm colloidal gold beads (Sigma-Aldrich, St. Louis, MO) precoated with bovine serum albumin. Four $\mu$ l of this mixture was applied onto freshly glow-discharged copper R2/2 Quantifoil holey carbon grids (Quantifoil Micro Tools GmbH, Jena, Germany). Using an FEI Vitrobot Mark IV, grids were then blotted (under 100\% humidity conditions) and plunge-frozen in a liquid ethane/propane mixture.

\section{Electron tomography and subtomogram averaging}

The frozen grids were subsequently imaged in an FEI Polara $300 \mathrm{keV}$ FEG transmission electron microscope (Thermo Fisher Scientific) coupled with a Gatan energy filter and a Gatan K2 Summit direct electron detector. Energy-filtered tilt series of cells were collected automatically from $-60^{\circ}$ to $+60^{\circ}$ at $1.5^{\circ}$ intervals using the UCSF Tomography data collection software ${ }^{53}$ with a cumulative total dosage of $100 \mathrm{e}^{-} \AA^{-2}$, a defocus of $-6 \mu \mathrm{m}$ and a pixel size of $3.9 \AA$. Using the IMOD software package ${ }^{54}$, the images were then binned by 2 , aligned and contrast transfer function corrected. Subsequently, SIRT reconstructions were produced using the TOMO3D program ${ }^{55}$. T2SS structures on cell envelopes were visually identified by their characteristic "hour-glass" like shape. Subtomogram averages of the $L$. pneumophila T2SS were generated by the PEET program ${ }^{56}$. The T2SS subtomogram averages exhibited a gross twofold symmetry around the central midline in the periplasm. Based on this observation, we applied a two-fold symmetry on the periplasmic complex. No symmetry was applied on the cytoplasmic complex. The T2SS exhibited a substantial flexibility between the OM- and IM-associated parts; binary masks were applied to perform focused alignments on the $\mathrm{OM}$ and on the IM complexes separately and finally combined to generate a composite structure. The numbers of tomograms collected and number of particles used are summarized in Supplementary Table 1.

\section{Identifying homologues between the L. pneumophila T2SS and the T4P system}

L. pneumophila Lp02 strain T2SS protein sequences were selected from the NCBI and UniProt databases. To identify corresponding components between the L. pneumophila T2SS and the MxT4aP and FcT4bP systems, we utilized two different programs: Phyre and Blast search ${ }^{35,57}$. This confirmed L. pneumophila T2SS components T2S C, D, E, F, G and $\mathrm{M}$ are homologues of the T4aP components PilP, PilQ, PilB, PilC, PilA and PilO respectively. Using a combination of Phyre, MOTIF-search, and Blast programs, we also predicted distinct domains and motifs within different T2SS components. 


\section{Building an architectural model of the intact $L$. pneumophila T2SS}

Recently, Chang et al used a combination of ECT, subtomogram averaging and genetic manipulations to determine the locations of all the major components in the MxT4aP and FcT4bP systems and produced an architectural model of the MxT4aP machinery ${ }^{33,34}$. However, in this case, because there is only about one T2SS particle in every -five $L$. pneumophila tomograms, a thorough mutant analysis (where individual components are either deleted or fused to a GFP) was not possible. Therefore, in order to assign the locations of T2SS components in our density map and build an architectural model of the T2SS, we performed a thorough sequence analysis and confirmed that the L. pneumophila T2SS components T2S C, D, E, F, G, and M are homologues of the T4aP components PilP, PilQ, PilB, PilC, PilA and PilO respectively ${ }^{58}$. And used this information to building an architectural model of the intact $L$. pneumophila T2SS.

Interestingly, the cytoplasmic domain of $\mathrm{T} 2 \mathrm{~S} \mathrm{~L}$ is homologous to the $\mathrm{T} 4 \mathrm{aP}$ component PilM (Phyre: 30\% coverage, 97\% confidence and 15\% identity) and the periplasmic domain of $\mathrm{T} 2 \mathrm{~S} \mathrm{~L}$ is homologous to the T4aP component PilN (Phyre: 23\% coverage, 78.6\% confidence and 13\% identity) implying T2S L is a fusion of the T4aP proteins PilM and PilN as previously suggested in other systems ${ }^{59}$.

Since atomic models are available for all the soluble domains of the T2SS components ${ }^{9,10}$, we sought to build an architectural model of the intact T2SS guided by the T4aP model (Fig. 3). The L. pneumophila T2SS has a total of 12 components. We began by placing an atomic model of the OM protein T2S D/secretin (PDB ID: 5WQ8) in our density map. The position of T2S D along its axis (perpendicular to the cell envelope) was set by aligning the gate densities. The N1, N2 and N3 domains of 5WQ8 matched well with the subtomogram average density map (Extended Data Fig. 3A-C). While the NO domain of secretin was not resolved in most of the previously reported single particle reconstructions, presumably due to flexibility, in our subtomogram average we saw an additional density for the NO domain just below N1 (Extended Data Fig. 3A-C) as recently seen by Chernyatina et al ${ }^{17}$. Our interpretation is that the $\mathrm{NO}$ domain is stabilized by the presence of its connecting subcomplexes and the rest of the cellular envelope. A co-crystal structure of the T2S D NO domain and the C-terminal homology region (HR) domain of the clamp protein T2S C (PDB ID: 3OSS) was therefore placed in this density below the N1 domain. Since no oligomeric structure is available for T2S C, we used known information about symmetry, connectivities and orientation (e.g. towards membrane etc.) to first generate numerous 15-mer (the known symmetry of T2S D) ring models for 3OSS using SymmDock ${ }^{60}$ and placed the ring model in the electron density map that fit the density best and satisfied all other criteria. Recently, Chernyatina et al showed that Klebsiella pneumoniae T2S CELM constitute a 2:1:1:1 complex and 6-12 (or maybe up to 15) copies of T2S C are present in this assembly (personal communication) ${ }^{17}$. Therefore, we removed 9 copies of T2S C randomly around the ring in the model to reflect this lowest stoichiometry and emphasize that not all T2S D's are bound by a $\mathrm{T} 2 \mathrm{~S} \mathrm{C}^{17}$. Other than the C-terminal HR domain, L. pneumophila $\mathrm{T} 2 \mathrm{~S} \mathrm{C}$ contains a periplasmic disordered region and an $\mathrm{N}$-terminal transmembrane helix. We modeled the disordered region of T2S C and placed the N-terminal helix in the IM. 
The periplasmic domains of T2S L (PDB ID: 2W7V) and M (PDB ID: 1UV7) both fold into ferredoxin-like domains and are known to bind each other ${ }^{41}$. We used a T2S M dimer structure (PDB ID: 1UV7) to first generate a T2S ML heterodimer and then used SymmDock to produce several hexameric ring models of the T2S ML heterodimer. We selected the model that best matched the lower-periplasmic ring-density. To build a working model for the pseudopilus, we used a cryoEM structure of the major pseudopilin filament (PDB ID: 5WDA), a co-crystal structure of the minor pseudopilin complex T2S IJK (PDB ID: 3CI0) and a crystal structure of the minor pseudopilin T2S H (PDB ID: 2KNQ) and their known connectivities and interfaces ${ }^{12,22,61}$. Since in our tomograms we only found pseudopili extending from the IM to the base of the secretin channel, we found that $\sim 5$ copies of major pseudopilin (T2S G) and one copy of each of the minor pseudopilins (T2S I, $\mathrm{J}, \mathrm{K}, \mathrm{H})$ were sufficient to extend this distance.

To begin to generate a working model for the cytoplasmic complex, we modelled a T2S F dimer structure based on the proposed structure of the $\mathrm{T} 4 \mathrm{aP}$ homologue $\mathrm{PilC}^{34}$ and placed this model in the cytoplasmic dome density. The T2SS ATPase T2S E has three distinct domains (N-terminal domains N1E, N2E and a C-terminal ATPase domain CTE) connected by two extended flexible linkers. We used a hexameric structure of the N2E+CTE domains of T2S E (PDB ID: 4KSS) and a co-crystal structure of the cytoplasmic domain of T2S L and the N1E domain of T2S E (PDB ID: 4PHT) to build working models for the cytoplasmic disk and ring densities, respectively. The cytoplasmic ring is $20 \mathrm{~nm}$ wide in diameter in our average. Given the experimentally determined stoichiometry of six T2S L molecules per secretin channel, we first used SymmDock to generate candidate ring assemblies of six 4PHT complexes. Interestingly, out of 5000 candidate ring models, none had a diameter more than $17 \mathrm{~nm}$. Our interpretation of this result is that the T2S L:N1E complex does not form a continuous ring in situ. Rather we simply placed six separate copies of 4PHT around the cytoplasmic ring with gaps in between. The 4KSS structure fits nicely into the cytoplasmic disk as expected. Finally, T2S O is an IM peptidase that processes the pseudopilins prior to their assembly. This protein has no significant domain outside the membrane and is not visible in our subtomogram average. Therefore, we did not place this protein in our architectural model.

\section{Extended Data}




\begin{tabular}{|c|c|c|c|c|}
\hline General description & Lp_T2SS & $M x_{\perp} T A Q P$ & $V c_{-} T 4 b P$ & Comments \\
\hline Secretin & LspD & PAQ & TepC & $\begin{array}{l}\text { Secretin forms a periplasmic vestibule and OM pore. } \\
\text { The T2SS vestibule is much longer than the T4aP/T4hP vestibule due to its multiple N-domains and ladks a distinct upper ring. } \\
\text { A longer secretin channel in the T2SS may facilit tate effector loading and translocation. }\end{array}$ \\
\hline \multirow[t]{2}{*}{ Pilotin } & & Tsap & $\operatorname{TapQ}$ & $\begin{array}{l}\text { Pllotins assist in the assembly and stabilization of the secretin channel. } \\
\text { TsaP binds to peptidoglycan and perhaps provides additional stability to the secretin. } \\
\text { TcpQ is required for localization and stability of its secretin. }\end{array}$ \\
\hline & & & & $\begin{array}{l}\text { Legionelita I2SS has no pilotin. Also, LspD has no obvious membrane targeting sequence. } \\
\text { How the LPTZSS targets to and assembles at the OM is undear. }\end{array}$ \\
\hline Clamp-protein & LspC & PilP & Teps & 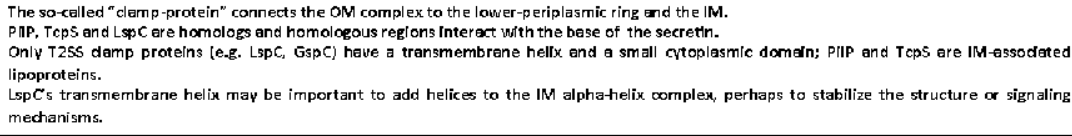 \\
\hline \multirow[t]{3}{*}{$\begin{array}{l}\text { Lower-periplasmic } \\
\text { ring }\end{array}$} & $\begin{array}{c}\text { LspL's } \\
\text { periplasmic } \\
\text { domain, LspM }\end{array}$ & PilN, Pilo & $T \mathrm{TPD}$ & 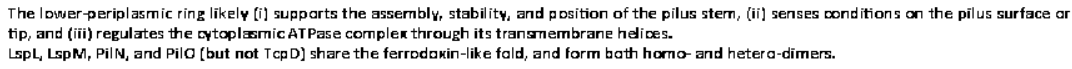 \\
\hline & & & & $\begin{array}{l}\text { In the T4aP system, } 12 \text { or more periplasmic domains of both PilN and PilO form what is likely two dose-packed rings. } \\
\text { In the T2SS, } 5 \mathrm{LspL} \text { and } 6 \mathrm{LspM} \text { periplasmic domains form what appears to be one ring. Cargo binding promotes formation of heterodimers, so }\end{array}$ \\
\hline & & & & $\begin{array}{l}\text { the interplay between homo-and hetero-dimers may be involved in signaling, as in the T4aP system. } \\
\text { In T4bP, TcpD alone forms the lower periplasmic ring, and it may not have a signaling function, since TabP pili are not actively retracted. } \\
\text { All these lower-periplasmic-ring proteins have transmembrane helices, which together form another ring in the IM. }\end{array}$ \\
\hline \multirow[t]{2}{*}{$\begin{array}{l}\text { AT Paso docking ring } \\
\text { (Crtoplasmic ring) }\end{array}$} & $\begin{array}{l}\text { Lspl's } \\
\text { ertoplasmie } \\
\text { domain }\end{array}$ & PIIM & TepR & $\begin{array}{l}\text { The ATPase docking ring recrults the A.TPase complex to the edapter. } \\
\text { The cyteplasmic domain of Lsp Lis homelogous to PilM, so LspL is a fusion of the T4aP proteins PilM and PilN. } \\
\text { The LspL ring is not continuous, as there are apparently only } 5 \text { monemers. }\end{array}$ \\
\hline & & & & $\begin{array}{l}\text { In the T2SS, the A.TPase docking ring appears to "clutch" the ATPase on and off for rapid crdes of growth and retraction. } \\
\text { In the T4aF, the ATP ase docking ring appears to select between growth and retraction ATP ases, and hold either one stably in place. }\end{array}$ \\
\hline Adapter complex & LspF & PilC & TCPE & $\begin{array}{l}\text { Recruits pilin/pseudopilin subunits during pilus/pseud opilus growth. } \\
\text { Mediates energy transfer from the ATPase complex to the pil us/pseudopilus, likely through an Archimedian-screws-like rotation. }\end{array}$ \\
\hline Mejor pilin & LspG & PilA & TOPA & Mejor subunit for the pilus / pseudop ilus structure. \\
\hline Minor pilins & LspIJKH & $\begin{array}{c}\text { Pilv (1-3), } \\
\text { PilW }(1-3) \\
\text { Fimu }(1-3) \\
\text { PilX] }\end{array}$ & TqB & $\begin{array}{l}\text { Minar-pseudopi ins assemble into a stable complex that interacts with the lower periplasmic ring. } \\
\text { The T25S has four minor pilins, the T4aP has three different and independent sets of four, and the T4bP system has only one. }\end{array}$ \\
\hline \multirow[t]{2}{*}{$\begin{array}{l}\text { AT Pase complex } \\
\text { (Cytoplasmic disk) }\end{array}$} & LspE & PilB, PilT & TCpT & $\begin{array}{l}\text { The ATPase powers pilus growth and sometimes retraction (în the T4aP system), likely by rotating the adapter. } \\
\text { The T2SS ATPase Lsp has three distinct domains (N1E, N2E and CTE) connected by two flexible linkersi the T4a/bP assernbly ATPases PilB/T CPT } \\
\text { lack any N1E domain. }\end{array}$ \\
\hline & & & & The T4aP system has a retraction AT Pase (PilT) but T2SS and T4bP systems have none. \\
\hline Prepilin peptidase & Lspo & PîlD & TсpJ & aves the leader sequences from prepilin and facil itates pilus/pseudopilus assembly. \\
\hline
\end{tabular}

Extended Data Fig. 1:

Comparison between the $\mathrm{T} 2 \mathrm{SS}$ and related molecular machines $\mathrm{T} 4 \mathrm{aP}$ and $\mathrm{T} 4 \mathrm{bP}$ systems. 

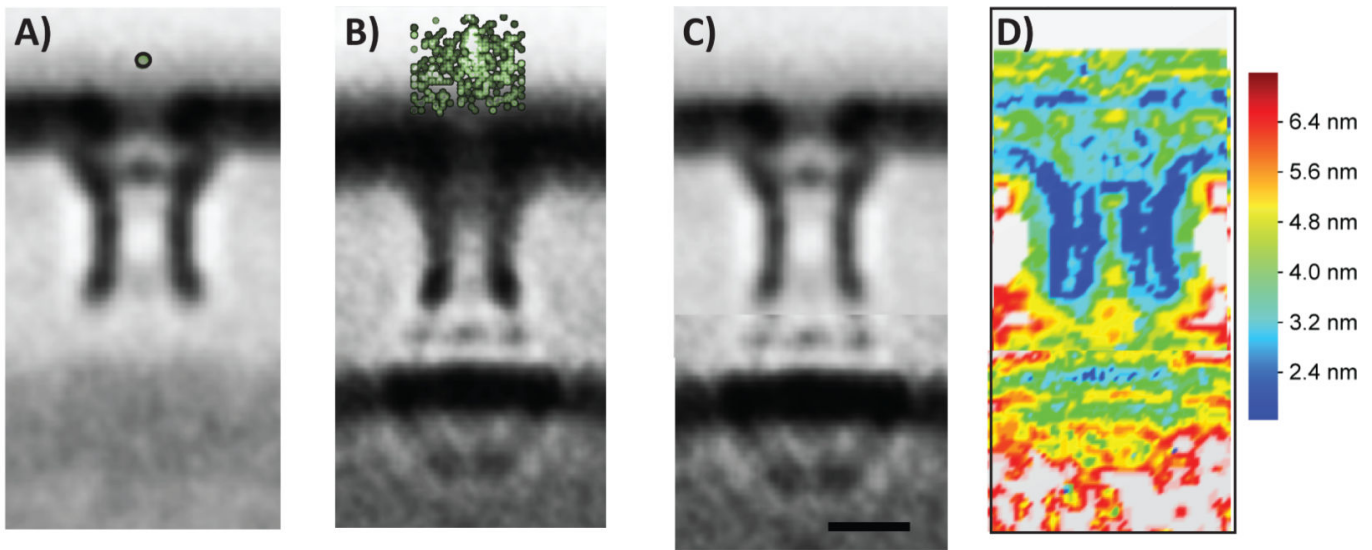
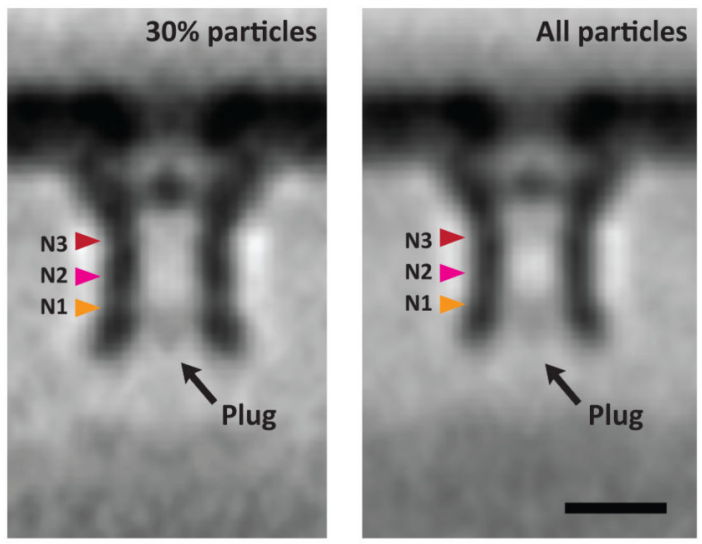

F)
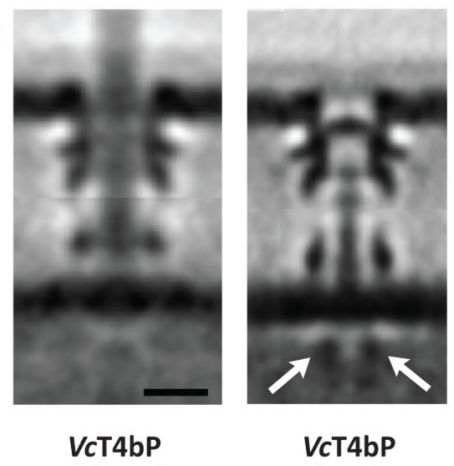

Piliated

$\triangle t c p R$

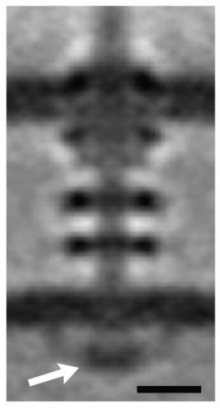

MxT4aP

Piliated

Extended Data Fig. 2. T2SS Flexibility.

Subtomogram averages of all particles aligned on (A) the OM-associated complex and (B) the IM-associated complex. The distribution of the green dots in (B) indicates the translations imposed on the OM complexes to align the IM complexes. (C) A composite average using the upper and lower halves of (A) and (B), respectively. D) Local resolution of (C) calculated by Resmap. (E) Focused alignment near the base of the secretin channel revealed the presence of a plug-like structure. $20 \%$ of the particles with highest crosscorrelation showed this distinct density. In the rest of the particles, the plug density is either not present or so dynamic that including them makes the plug almost invisible. (F) 
Previously reported in situ averages of the T4aP (WT, piliated) and T4bP (WT, piliated and $\Delta t c p R$ mutant) machines in states with cytoplasmic dome, ring and disks for comparison ${ }^{33,34}$. White arrows indicate cytoplasmic disks. Scale bars, $10 \mathrm{~nm}$ (A-C), (E), (F). 

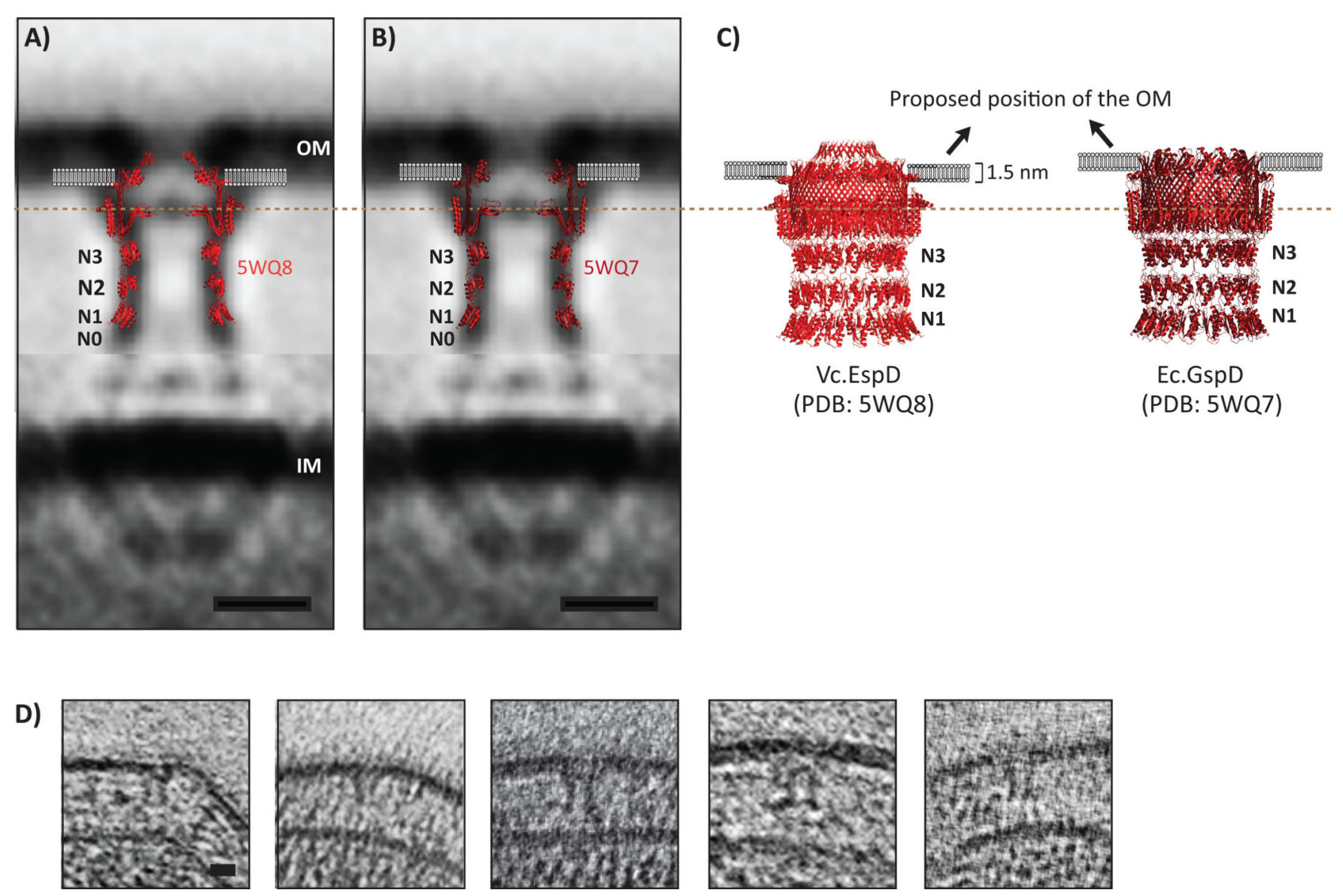

Extended Data Fig. 3. Position of the T2SS secretin with respect to the OM.

(A and B) Atomic models of the $V$. cholerae (PDB ID: 5WQ8) and E. coli (PDB ID: 5WQ7)

T2SS secretins superimposed on our subtomogram average based on the position of the gate.

(C) Positions of the OM on these structures as suggested in earlier publications ${ }^{13,15}$. The widths of the suggested OM spanning regions were only $\sim 1.8 \mathrm{~nm}$, but real membranes are known to be 5-7 $\mathrm{nm}$ wide. In all reported atomic models, the secretin channel is suggested to extend beyond the $\mathrm{OM}^{13,9}$. However, when we overlaid the secretin atomic models on our subtomogram average, it only reached through the inner leaflet of the OM. Scale bars, 10 nm. D) Tomographic slices of mutant L. pneumophila cells lacking all major and minor pilins $(\Delta / s p G H I J K)$. Showing representative individual T2SS particles. No pseudopilus or lower-periplasmic ring is visible. A similar result was obtained when we examined a $L$. pneumophila $\Delta$ spHIJK mutant. Scale bar, $10 \mathrm{~nm}$ (D). For each strain, number of tomograms recorded and number of particles found are listed in the SI Table-1. 

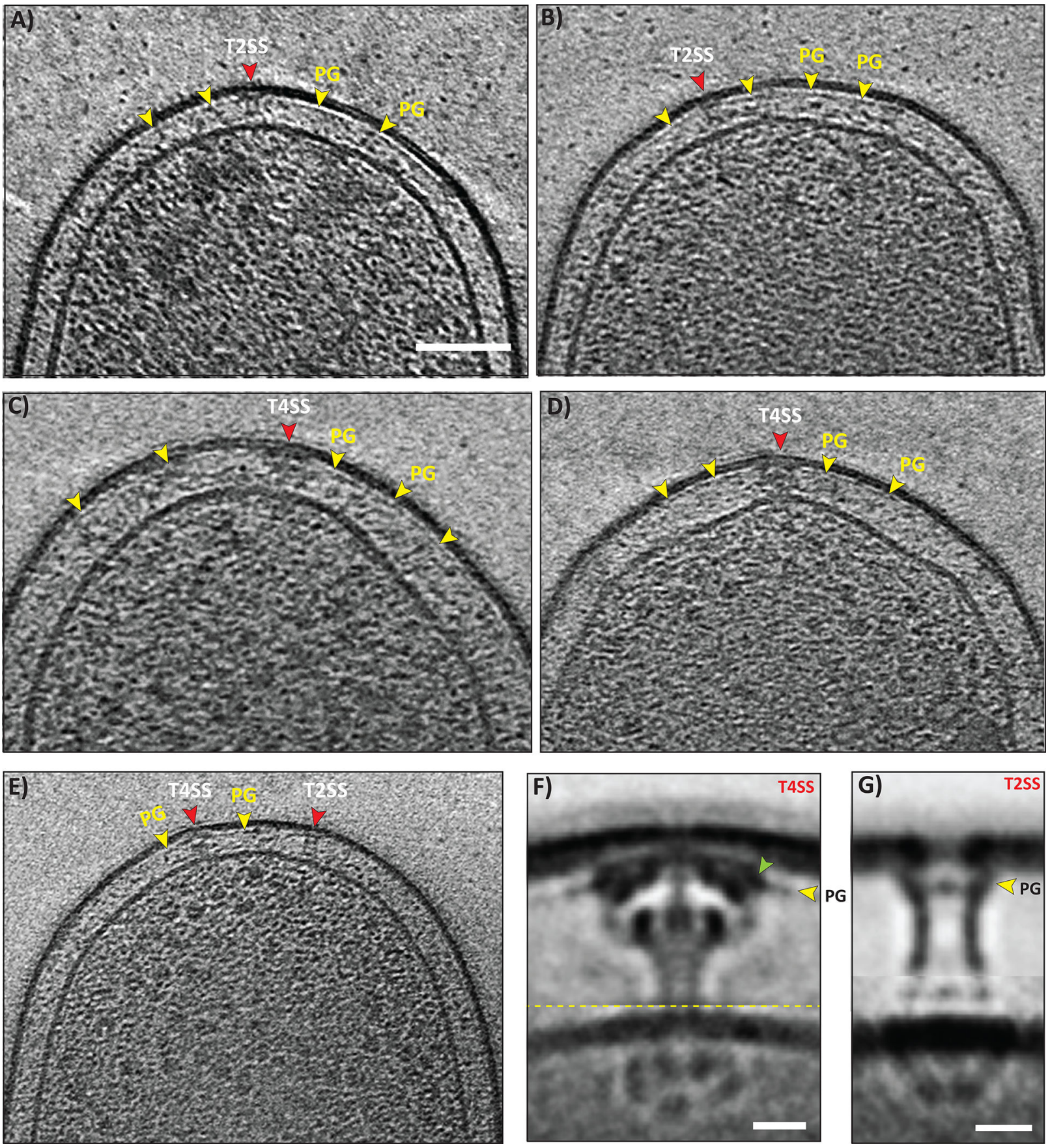

Extended Data Fig. 4. Position of the T2SS with respect to the PG layer in L. pneumophila.

(A and B) Tomographic slices through $L$. pneumophila cells showing T2SS particles (red arrowheads) and the peptidoglycan layer (PG, yellow arrowheads). (C and D) Tomographic slices through $L$. pneumophila cells showing T4BSS particles (red arrowheads) and the peptidoglycan layer (PG, yellow arrowheads). (E) Tomographic slice through a $L$. pneumophila cell showing both T4SS and T2SS particles (red arrowheads) and peptidoglycan (PG, yellow arrowheads) in the same cell. (F and G) Subtomogram averages of the T4SS and T2SS, respectively. DotK (shown as green arrow) in the T4BSS is known to 
interact with the PG layer confirming its location just a few nm below the OM (F). We therefore conclude that the PG layer surrounds the T2SS at approximately the level of the gate $(G)$. For each strain, number of tomograms recorded and number of particles found are listed in the SI Table-1. Scale bars, $100 \mathrm{~nm}$ (A-E), $10 \mathrm{~nm}$ (F-G). 
Extended Data Fig. 5. Cryo-EM data collection, refinement and validation statistics

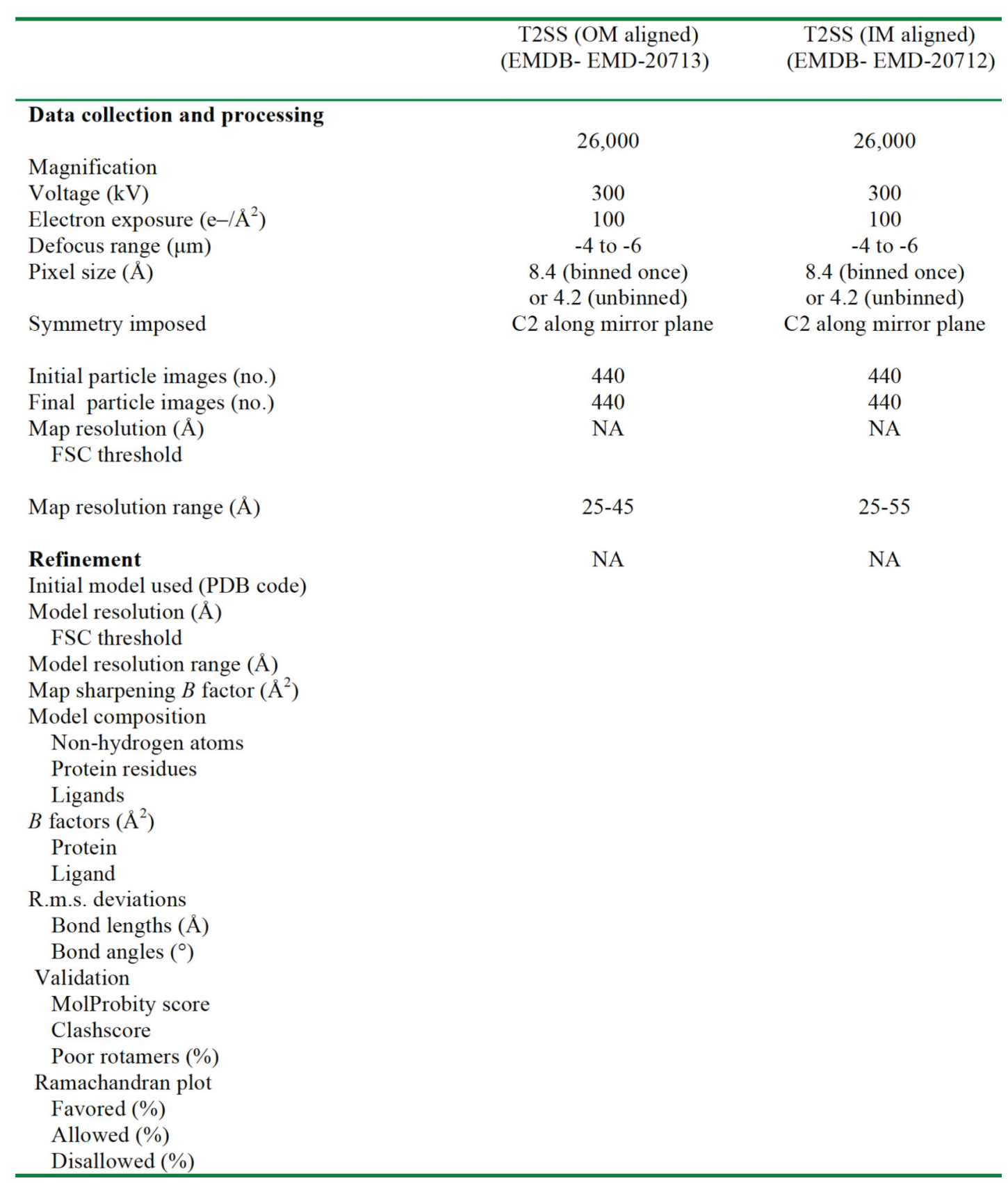

Extended Data Fig. 5.

Cryo-EM data collection, refinement and validation statist

\section{Supplementary Material}

Refer to Web version on PubMed Central for supplementary material. 


\section{Acknowledgements}

This work was supported by NIH grants AI127401 to G.J.J. and AI043987 to N.P.C. ECT data were recorded at the Beckman Institute Resource Center for Transmission Electron Microcopy at Caltech and the cryo-EM facility at Janelia Research Campus. We thank Drs. Davi Ortega, Yi- Wei Chang and Richard C. White for helpful discussions. M. K. is supported by a postdoctoral Rubicon fellowship from De Nederlandse Organisatie voor Wetenschappelijk Onderzoek (NWO).

\section{References}

1. Costa TRD et al. Secretion systems in Gram-negative bacteria: structural and mechanistic insights. Nat. Rev. Microbiol 13, 343-359 (2015) [PubMed: 25978706]

2. Abby SS et al. Identification of protein secretion systems in bacterial genomes. Sci. Rep 6, 23080 (2016). [PubMed: 26979785]

3. White RC \& Cianciotto NP Assessing the impact, genomics and evolution of type II secretion across a large, medically important genus: the Legionella type II secretion paradigm. Microb Genom, 5(6): e000273 (2019).

4. Hospenthal MK, Costa TRD \& Waksman G A comprehensive guide to pilus biogenesis in Gramnegative bacteria. Nat. Rev. Microbiol 15, 365-379 (2017). [PubMed: 28496159]

5. Peabody CR et al. Type II protein secretion and its relationship to bacterial type IV pili and archaeal flagella. Microbiology (Reading, Engl.) 149, 3051-3072 (2003).

6. Cianciotto NP Type II secretion: a protein secretion system for all seasons. Trends Microbiol. 13, 581-588 (2005). [PubMed: 16216510]

7. Cianciotto NP \& White RC Expanding Role of Type II Secretion in Bacterial Pathogenesis and Beyond. Infect. Immun 85, e00014-17, (2017). [PubMed: 28264910]

8. Thomassin J-L, Santos Moreno J, Guilvout I, Tran Van Nhieu G \& Francetic O The trans-envelope architecture and function of the type 2 secretion system: new insights raising new questions. Mol. Microbiol 105, 211-226 (2017). [PubMed: 28486768]

9. Filloux A \& Voulhoux R Multiple Structures Disclose the Secretins' Secrets. J. Bacteriol 200, p. 914 (2018).

10. Nivaskumar M \& Francetic O Type II secretion system: A magic beanstalk or a protein escalator. Biochimica et Biophysica Acta (BBA) - Molecular Cell Research 1843, 1568-1577 (2014). [PubMed: 24389250]

11. Nunn D Bacterial type II protein export and pilus biogenesis: more than just homologies? Trends Cell Biol. 9, 402-408 (1999). [PubMed: 10481178]

12. Lopez-Castilla A et al. Structure of the calcium-dependent type 2 secretion pseudopilus. Nat. Microbiol 2, 1686-1695 (2017). [PubMed: 28993624]

13. Yan Z, Yin M, Xu D, Zhu Y \& Li X Structural insights into the secretin translocation channel in the type II secretion system. Nat. Struct. Mol. Biol 24, 177-183 (2017). [PubMed: 28067918]

14. Hay ID, Belousoff MJ, Dunstan RA, Bamert RS \& Lithgow T Structure and Membrane Topography of the Vibrio-Type Secretin Complex from the Type 2 Secretion System of Enteropathogenic Escherichia coli. Journal of Bacteriology 200, (2017).

15. Yin M, Yan Z \& Li X Structural insight into the assembly of the type II secretion system pilotinsecretin complex from enterotoxigenic Escherichia coli. Nat. Microbiol 3, 581-587 (2018). [PubMed: 29632366]

16. Howard SP et al. Structure and assembly of pilotin-dependent and -independent secretins of the type II secretion system. PLoS Pathog. 15, e1007731 (2019). [PubMed: 31083688]

17. Chernyatina AA \& Low HH Architecture of a bacterial type II secretion system. bioRxiv (2018). doi:10.1101/397794

18. Hay ID, Belousoff MJ \& Lithgow T Structural Basis of Type 2 Secretion System Engagement between the Inner and Outer Bacterial Membranes. MBio 8, (2017).

19. Yamagata A \& Tainer JA Hexameric structures of the archaeal secretion ATPase GspE and implications for a universal secretion mechanism. EMBO J. 26, 878-890 (2007). [PubMed: 17255937] 
20. Lu C et al. Hexamers of the type II secretion ATPase GspE from Vibrio cholerae with increased ATPase activity. Structure 21, 1707-1717 (2013). [PubMed: 23954505]

21. Korotkov KV et al. Structural and functional studies on the interaction of GspC and GspD in the type II secretion system. PLoS Pathog. 7, e1002228 (2011). [PubMed: 21931548]

22. Korotkov KV \& Hol WGJ Structure of the GspK-GspI-GspJ complex from the enterotoxigenic Escherichia coli type 2 secretion system. Nat. Struct. Mol. Biol 15, 462-468 (2008). [PubMed: 18438417]

23. Zhang Y et al. Structure-guided disruption of the pseudopilus tip complex inhibits the Type II secretion in Pseudomonas aeruginosa. PLoS Pathog. 14, e1007343 (2018). [PubMed: 30346996]

24. Abendroth J, Rice AE, McLuskey K, Bagdasarian M \& Hol WGJ The crystal structure of the periplasmic domain of the type II secretion system protein EpsM from Vibrio cholerae: the simplest version of the ferredoxin fold. J. Mol. Biol 338, 585-596 (2004). [PubMed: 15081815]

25. Abendroth J, Kreger AC \& Hol WGJ The dimer formed by the periplasmic domain of EpsL from the Type 2 Secretion System of Vibrio parahaemolyticus. J. Struct. Biol 168, 313-322 (2009). [PubMed: 19646531]

26. Abendroth $\mathrm{J}$ et al. The three-dimensional structure of the cytoplasmic domains of EpsF from the type 2 secretion system of Vibrio cholerae. J. Struct. Biol 166, 303-315 (2009). [PubMed: 19324092]

27. Ghosal D et al. Molecular architecture, polar targeting and biogenesis of the Legionella Dot/Icm T4SS. Nat. Microbiol 4, 1173-1182 (2019). [PubMed: 31011165]

28. Kaplan $\mathrm{M}$ et al. The presence and absence of periplasmic rings in bacterial flagellar motors correlates with stator type. Elife 8, e43487 (2019). [PubMed: 30648971]

29. Ghosal D, Kaplan M, Chang Y-W \& Jensen GJ In situ imaging and structure determination of bacterial toxin delivery systems using electron cryotomography. Methods Mol. Biol 1921, 249265 (2019). [PubMed: 30694497]

30. Oikonomou CM, Chang Y-W \& Jensen GJ A new view into prokaryotic cell biology from electron cryotomography. Nat. Rev. Micro 14, 205-220 (2016).

31. Hu B et al. Visualization of the type III secretion sorting platform of Shigella flexneri. Proc. Natl. Acad. Sci. U.S.A 112, 1047-1052 (2015). [PubMed: 25583506]

32. Liles MR, Viswanathan VK \& Cianciotto NP Identification and temperature regulation of Legionella pneumophila genes involved in type IV pilus biogenesis and type II protein secretion. Infect. Immun 66, 1776-1782 (1998). [PubMed: 9529113]

33. Chang Y-W et al. Architecture of the Vibrio cholerae toxin-coregulated pilus machine revealed by electron cryotomography. Nat. Microbiol 2, 16269 (2017). [PubMed: 28165453]

34. Chang Y-W et al. Architecture of the type IVa pilus machine. Science 351, aad2001 (2016). [PubMed: 26965631]

35. Kelley LA, Mezulis S, Yates CM, Wass MN \& Sternberg MJE The Phyre2 web portal for protein modeling, prediction and analysis. Nat. Protoc 10, 845-858 (2015). [PubMed: 25950237]

36. Adebali O, Ortega DR \& Zhulin IB CDvist: a webserver for identification and visualization of conserved domains in protein sequences. Bioinformatics 31, 1475-1477 (2015). [PubMed: 25527097]

37. D'Imprima E et al. Cryo-EM structure of the bifunctional secretin complex of Thermus thermophilus. Elife 6, e30483 (2017). [PubMed: 29280731]

38. Cisneros DA, Bond PJ, Pugsley AP, Campos M \& Francetic O Minor pseudopilin self-assembly primes type II secretion pseudopilus elongation: Role of minor pseudopilins in type II secretion. The EMBO Journal 31, 1041-1053 (2012). [PubMed: 22157749]

39. Gu S, Shevchik VE, Shaw R, Pickersgill RW \& Garnett JA The role of intrinsic disorder and dynamics in the assembly and function of the type II secretion system. Biochim Biophys Acta Proteins Proteom 1865, 1255-1266 (2017). [PubMed: 28733198]

40. Strozen TG et al. Involvement of the GspAB complex in assembly of the type II secretion system secretin of Aeromonas and Vibrio species. J. Bacteriol 193, 2322-2331 (2011). [PubMed: 21378198] 
41. Michel-Souzy S et al. Direct interactions between the secreted effector and the T2SS components GspL and GspM reveal a new effector-sensing step during type 2 secretion. J. Biol. Chem 293, 19441-19450 (2018). [PubMed: 30337370]

42. Abendroth J, Murphy P, Sandkvist M, Bagdasarian M \& Hol WGJ The X-ray structure of the type II secretion system complex formed by the N-terminal domain of EpsE and the cytoplasmic domain of EpsL of Vibrio cholerae. J. Mol. Biol 348, 845-855 (2005). [PubMed: 15843017]

43. Py B, Loiseau L \& Barras F An inner membrane platform in the type II secretion machinery of Gram-negative bacteria. EMBO Rep. 2, 244-248 (2001). [PubMed: 11266368]

44. Arts $\mathrm{J}$ et al. Interaction domains in the Pseudomonas aeruginosa type II secretory apparatus component XcpS (GspF). Microbiology (Reading, Engl.) 153, 1582-1592 (2007).

45. Waters RC, O'Toole PW \& Ryan KA The FliK protein and flagellar hook-length control. Protein Sci 16, 769-780 (2007). [PubMed: 17456739]

46. Jeong KC, Ghosal D, Chang Y-W, Jensen GJ \& Vogel JP Polar delivery of Legionella type IV secretion system substrates is essential for virulence. Proc. Natl. Acad. Sci. U.S.A 114, 8077-8082 (2017). [PubMed: 28696299]

47. Ghosal D, Chang Y-W, Jeong KC, Vogel JP \& Jensen GJ In situ structure of the Legionella Dot/Icm type IV secretion system by electron cryotomography. EMBO Rep. 18, 726-732 (2017). [PubMed: 28336774]

48. Vincent CD et al. Identification of the core transmembrane complex of the Legionella Dot/Icm type IV secretion system. Mol. Microbiol 62, 1278-1291 (2006). [PubMed: 17040490]

49. White RC et al. Type II Secretion-Dependent Aminopeptidase LapA and Acyltransferase PlaC Are Redundant for Nutrient Acquisition during Legionella pneumophila Intracellular Infection of Amoebas. MBio 9, e00528-18 (2018). [PubMed: 29666285]

50. Rossier O, Starkenburg SR \& Cianciotto NP Legionella pneumophila type II protein secretion promotes virulence in the A/J mouse model of Legionnaires' disease pneumonia. Infect. Immun 72, 310-321 (2004). [PubMed: 14688110]

51. Rossier O \& Cianciotto NP Type II protein secretion is a subset of the PilD-dependent processes that facilitate intracellular infection by Legionella pneumophila. Infect. Immun 69, 2092-2098 (2001). [PubMed: 11254562]

52. Truchan HK, Christman HD, White RC, Rutledge NS \& Cianciotto NP Type II secretion substrates of Legionella pneumophila translocate out of the pathogen-occupied vacuole via a semipermeable membrane. MBio 8, e00870-17 (2017). [PubMed: 28634242]

53. Zheng SQ et al. UCSF tomography: an integrated software suite for real-time electron microscopic tomographic data collection, alignment, and reconstruction. J. Struct. Biol 157, 138-147 (2007). [PubMed: 16904341]

54. Kremer JR, Mastronarde DN \& McIntosh JR Computer visualization of threedimensional image data using IMOD. J. Struct. Biol 116, 71-76 (1996). [PubMed: 8742726]

55. Agulleiro J-I \& Fernandez J-J Tomo3D 2.0--exploitation of advanced vector extensions (AVX) for 3D reconstruction. J. Struct. Biol 189, 147-152 (2015). [PubMed: 25528570]

56 . Nicastro $\mathrm{D}$ et al. The molecular architecture of axonemes revealed by cryoelectron tomography. Science 313, 944-948 (2006). [PubMed: 16917055]

57. Altschul SF et al. Gapped BLAST and PSI-BLAST: a new generation of protein database search programs. Nucleic Acids Res. 25, 3389-3402 (1997). [PubMed: 9254694]

58. Korotkov KV, Sandkvist M \& Hol WGJ The type II secretion system: biogenesis, molecular architecture and mechanism. Nat. Rev. Microbiol 10, 336-351 (2012). [PubMed: 22466878]

59. Leighton TL, Yong DH, Howell PL \& Burrows LL Type IV pilus alignment subcomplex proteins PilN and PilO form homo- and heterodimers in vivo. J. Biol. Chem 291, 19923-19938 (2016). [PubMed: 27474743]

60. Schneidman-Duhovny D, Inbar Y, Nussinov R \& Wolfson HJ PatchDock and SymmDock: servers for rigid and symmetric docking. Nucleic Acids Res. 33, W363-367 (2005). [PubMed: 15980490]

61. Douzi B et al. The XcpV/GspI pseudopilin has a central role in the assembly of a quaternary complex within the T2SS pseudopilus. J. Biol. Chem 284, 34580-34589 (2009). [PubMed: 19828448] 

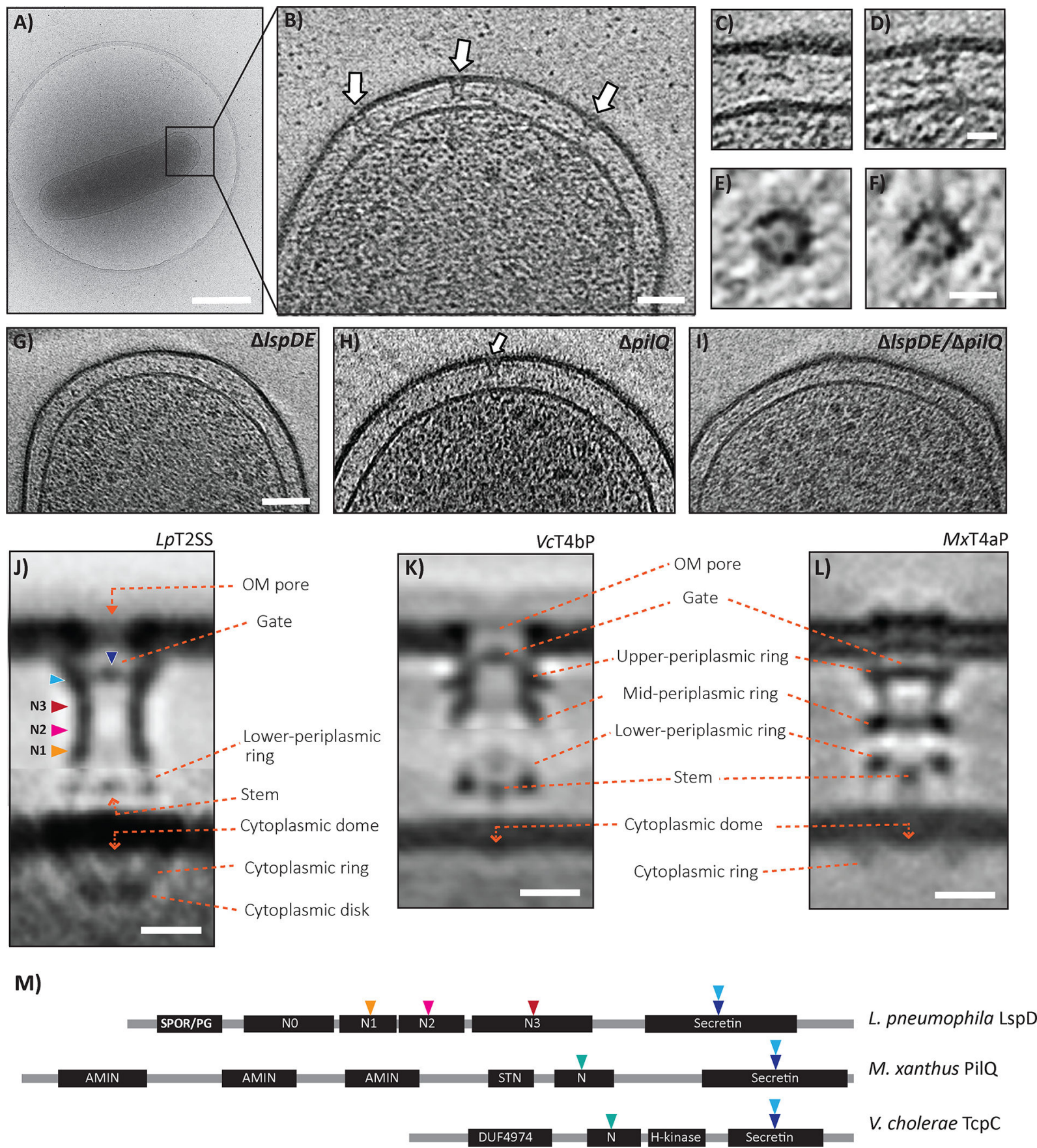

Fig. 1. Visualization of the type II secretion system (T2SS) in frozen-hydrated L. pneumophila cells.

(A) Cryo-EM image of a frozen-hydrated $L$. pneumophila cell on a Quantifoil grid. (B) Central slice through a tomographic reconstruction of a $L$. pneumophila cell pole. White arrows point to "hour-glass" shaped putative T2SS structures. (C and D) Tomographic slices showing individual T2SS particles. (E and F) Top views of individual T2SS particles. (G-I) Tomographic slices of L. pneumophila mutants lacking the T2SS secretin (G), the type IVa pili secretin (H), or both (I). (J) Composite subtomogram average of the wild-type $L$.

pneumophila T2SS. For each strain, number of tomograms recorded and number of particles 
found are listed in the SI Table-1. (K and L) Subtomogram averages of the Vibrio cholerae T4bP and Myxococcus xanthus $\mathrm{T}_{4} \mathrm{aP}^{33,34}$ for comparison. (M) Domain architectures of the secretin protein in the L. pneumophila T2SS (T2S D or LspD), M. xanthus T4aP (PilQ) and $V$. cholerae T4bP (TcpC). Protein regions corresponding to different densities are indicated by arrowheads. PG: Peptidoglycan binding domain, SPOR: Sporulation related repeat, STN: Secretin and TonB N terminus short domain. Scale bars, $500 \mathrm{~nm}$ (A), $50 \mathrm{~nm}$ (B), $20 \mathrm{~nm}$ (C, D), $10 \mathrm{~nm}(\mathrm{E}, \mathrm{F}), 50 \mathrm{~nm}(\mathrm{G}-\mathrm{I}), 10 \mathrm{~nm}(\mathrm{~J}-\mathrm{L})$. 

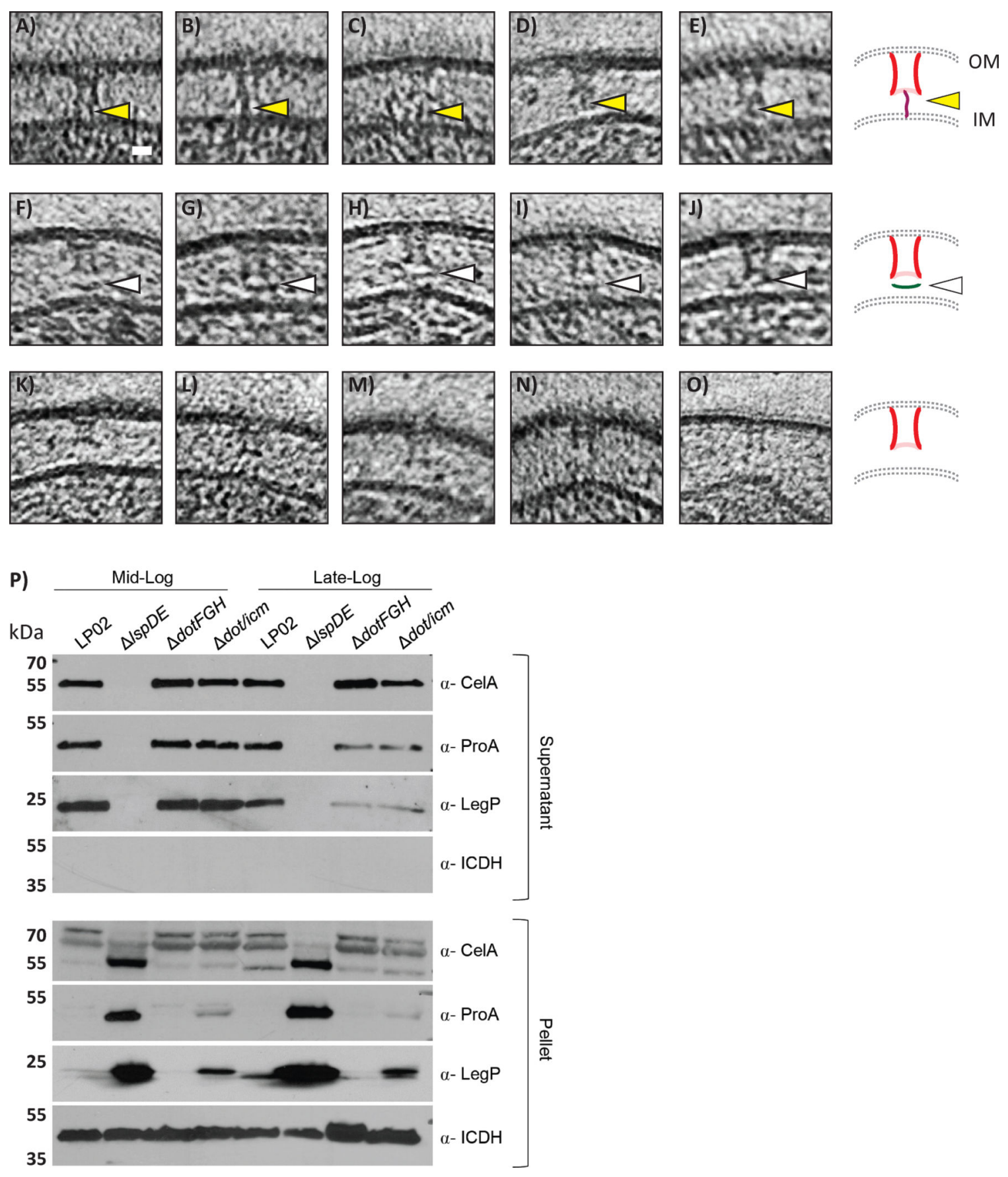

Fig. 2. Individual T2SS subcomplexes.

Tomographic slices showing individual T2SS particles with: (A-E) pseudopili (yellow arrowheads), (F-J) lower-periplasmic rings (white arrowheads) and (K-O) only secretin channels. Schematic representations (right). Scale bars, $10 \mathrm{~nm}$ (A-O). (P) Western blot analyses of T2SS effector (CelA, ProA and LegP) release in L. pneumophila culture supernatants at mid- and late-log phases. Different strains were analyzed: lanes- 1 and 5) WT L. pneumophila (Lp02), and mutant strains lacking 2 and 6) a functional T2SS $(\Delta l s p D E), 3$ and 7$)$ a functional Dot/Icm T4BSS ( $\Delta d o t H, \Delta d o t G$, and $\Delta d o t F:$ JV7058), 4 and 
8) all components of the Dot/Icm T4BSS (JV4044). The top set of immunoblots assesses the proteins in the culture supernatants, whereas the bottom set of immunoblots assesses the proteins within cell lysates. Blots assessing the presence of the cytoplasmic protein isocitrate dehydrogenase (ICDH) are presented as controls. In several rounds of experiments, we did not notice any consistent difference (increase or decrease) in T2SS effector release in Dot/Icm T4SS nonfunctional mutants. Shown here are representative western blots. Experiments were done in duplicates and representative images are shown. 


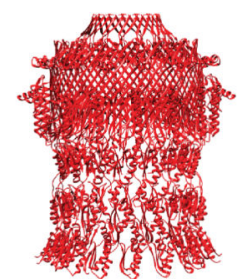

PDB: 5WQ8 (T2S D)
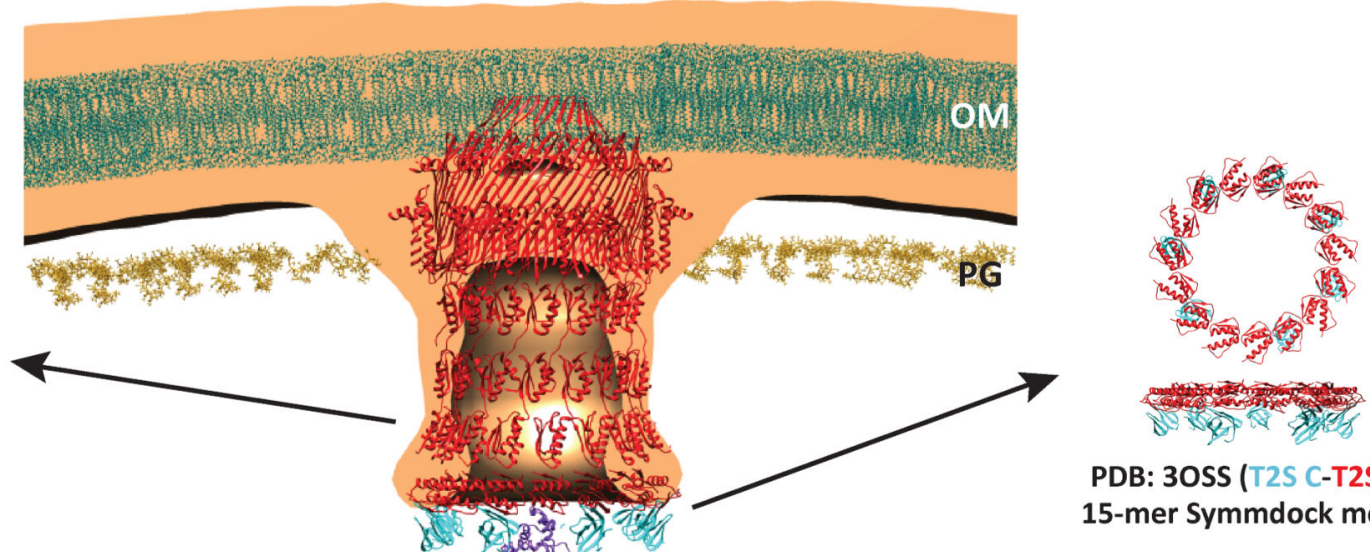

PDB: 30 SS (T2S C-T2S D)
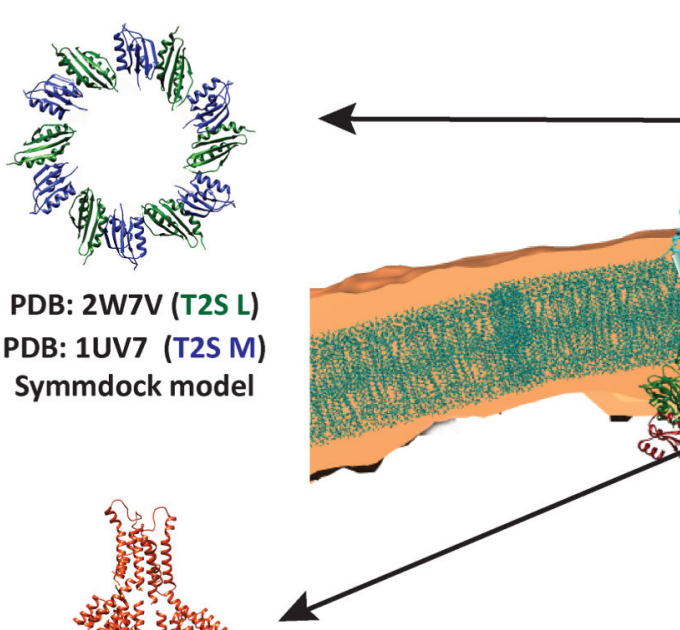

PDB: 2W7V (T2S L)

PDB: 1UV7 (T2S M)

Symmdock model

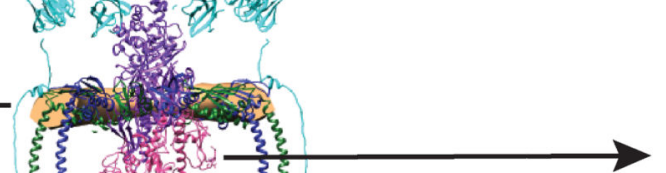

15-mer Symmdock model

PDB: $3 \mathrm{C} 1 \mathrm{Q}\left(\mathrm{T}^{2} \mathrm{SF}^{\mathrm{N}}\right)$

2WHN (T2S F $\left.{ }^{C}\right)$
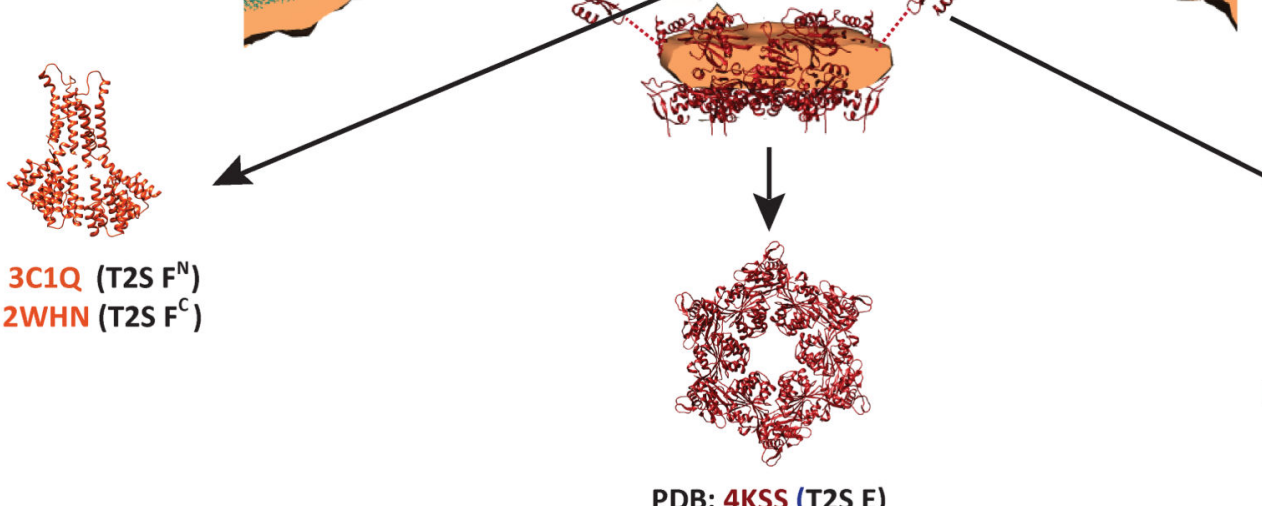

IM

PDB: 5WDA (major pilin)

PDB: 3 CIO (minor pilin T2S IJK)

PDB: 2KNQ (minor pilin T2S H)

PDB: 4KSS (T2S E)

Fig. 3. Architectural model of the T2SS.

Atomic models of T2SS components are superimposed on the central slice of the T2SS subtomogram average based on known connectivities and interfaces (see text).

Transmembrane domains of the IM proteins are shown as cylinders. Linkers between N1E domain and N2E/CTE domains of T2S E are represented by dotted lines. OM = outer membrane, $\mathrm{IM}=$ inner membrane, $\mathrm{PG}=$ peptidoglycan. Lipids are shown in dark cyan and peptidoglycan dark yellow. 